\title{
Heavy flavor/quarkonium production at the LHC
}

\author{
Hee Sok Chung* \\ Physik-Department, Technische Universität München, James-Franck-Str. 1, 85748 Garching, \\ Germany \\ E-mail: heesok. chung@tum.de
}

Inclusive one-hadron production cross sections provide valuable information about various aspects of QCD including perturbative and nonperturbative physics. New measurements at the LHC provide new opportunities to test our knowledge to new frontier. In order to obtain useful information from LHC measurements, it is important to have an understanding of hadron production mechanisms in hard collisions. Production cross sections of heavy flavored hadrons and heavy quarkonium contain the heavy quark mass as a hard scale, which lets us apply perturbation theory at the scale of the mass of the produced hadron. While this becomes an advantage when we want to take more theoretical control over hadron production mechanisms, approaches based on solid theoretical ground can sometimes become unsatisfactory. In this paper, we review the current status of heavy flavor and quarkonium production mechanisms that are popularly used to describe LHC data.

XXVII International Workshop on Deep-Inelastic Scattering and Related Subjects - DIS2019 8-12 April, 2019

Torino, Italy

${ }^{*}$ Speaker. 


\section{Introduction}

Theoretical investigation of production cross sections at the LHC provide probes of perturbative and nonperturbative QCD. Approaches based on the standard collinear factorization theorems $[1,2,3]$ can be used to gain information of the nonperturbative parton distribution functions (PDFs), while measurements at large momentum transfer can provide valuable tests of perturbative QCD. When initial-state partons have small collinear momenta, the cross sections can become sensitive to the transverse-momentum dependent (TMD) PDFs [4]. Hadrons produced in heavy ion collisions can be probes of the quark-gluon plasma.

Production cross sections of hadrons in hard processes are sensitive to the perturbative hard collision of quarks and gluons, and the configuration of the initial-state partons. While the hadronization process is essentially nonperturbative, the mass of the heavy quark provides an additional hard scale for production processes of heavy flavor and heavy quarkonia. For the case of heavy flavored meson production, calculations based on collinear factorization can be extended to cross sections at small transverse momenta [5]. Measurements at the LHC can provide tests of this formalism; for example, discrepancy between theory and data can be considered a probe of the TMD PDFs.

The situation is a bit more complicated in the case of heavy quarkonia, which are bound states of a heavy quark $Q$ and a heavy antiquark $\bar{Q}$. When the heavy-quark mass is much larger than $\Lambda_{\mathrm{QCD}}$, the production cross section may factorize into the perturbative production of the $Q \bar{Q}$ pair, followed by the nonperturbative formation of the heavy quarkonium from the $Q \bar{Q}$. It is yet unclear whether this is a valid approach, and the experimental determination of the nonperturbative hadronization process is still unresolved. Once this production mechanism is established, the production cross sections can be used to explore new areas of QCD, like the formation of heavy quarkonium in a hot and dense media.

The remainder of this paper is organized as follows. We review production mechanisms of heavy flavored mesons in Sec. 2. In Sec. 3, we discuss proposed mechanisms of heavy quarkonia, including effective field theory based approaches and models. We conclude in Sec. 4.

\section{Heavy flavor production}

Production of heavy flavor in hard collisions can be described by heavy quark fragmentation [5]. In this formalism, production cross section of a $B$ meson in lepton colliders is given by

$$
\sigma\left(e^{+} e^{-} \rightarrow B+X\right)=\hat{\sigma}\left(e^{+} e^{-} \rightarrow b+X\right) \otimes D_{b \rightarrow B},
$$

where $\hat{\sigma}\left(e^{+} e^{-} \rightarrow b+X\right)$ is the perturbative cross section of a $b$ quark, $D_{b \rightarrow B}$ is the nonperturbative fragmentation function, and $\otimes$ is a convolution given by the integral over the fractional momentum of the $B$ meson compared to the momentum of the $b$ quark. At transverse momenta much larger than the $b$ quark mass, the $b$ quark mass can be ignored, and the effects of parton splitting must be included; then, the formula (2.1) becomes the standard one in collinear factorization [6]. The nonperturbative fragmentation function is a universal quantity; they can be determined by comparing Eq. (2.1) with lepton collider experiments. Then, they can be used to predict production cross sections at hadron colliders. In proton-proton collisions, the cross section can be written as

$$
\sigma(p p \rightarrow B+X)=\sum_{i j} f_{i / p} \otimes f_{j / p} \otimes \hat{\sigma}(i j \rightarrow b+X) \otimes D_{b \rightarrow B},
$$


where $f_{i / p}$ is the proton PDF, and $D_{b \rightarrow B}$ is the same nonperturbative fragmentation function appearing in Eq. (2.1). The same formalism applies to the production of $D$ mesons, where the $b$ quarks in Eqs. (2.1) and (2.2) are replaced by the $c$ quark. This formalism has been used to predict $B$ and $D$ meson cross sections at the LHC. Specific implementations of the formalism include the widely used "FONLL" calculation [7, 8], where the fixed-order calculation of the perturbative cross section is included to next-to-leading order (NLO) accuracy in the strong coupling, and the evolution of the fragmentation function at large transverse momentum have been included to next-to-leading logarithmic (NLL) accuracy. In Ref. [9], a similar calculation has been done in the general-mass variable-flavor-number (GM-VFN) scheme. These calculations are in very good agreement with recent LHC measurements of $B$ and $D$ meson cross sections $[10,11]$. The precision of LHC measurements now surpass theoretical uncertainties, and calls for more theoretical effort to catch up.

Since the production mechanism is in good theoretical control, $B$ and $D$ meson production may be a good place to look for effects of TMD PDFs and medium effects. Explicit calculations show that using the TMD formalism has a small effect on the cross section at small transverse momenta [12], so that more precision in both theory and experiment are needed to quantify such effects in heavy flavor production. Measurements of heavy flavored hadron cross sections in proton and heavy-ion collisions are in good agreement with current understanding of cold nuclear matter effects [13].

\section{Heavy quarkonium production}

Most theoretical studies on heavy quarkonium production involve an assumption that the production cross section factorizes into the perturbative cross section of a $Q \bar{Q}$ pair, followed by the nonperturbative evolution of the $Q \bar{Q}$ to the quarkonium state. There are approaches based on nonrelativistic effective field theories of QCD, such the nonrelativistic QCD (NRQCD) [14]. Unfortunately, phenomenological studies based on NRQCD sometimes lead to unsatisfactory description of heavy quarkonium production data in colliders. For this reason, models such as the color-singlet model or the color-evaporation model are sometimes used. In this section, we give a brief overview of these popularly used formalisms.

\subsection{Nonrelativistic QCD}

NRQCD provides a factorization formula that describes inclusive quarkonium production cross sections as [14]

$$
\sigma(H+X)=\sum_{n} \hat{\sigma}(Q \bar{Q}(n)+X)\left\langle 0\left|\mathscr{O}_{n}^{H}\right| 0\right\rangle,
$$

where $H$ is a quarkonium, $\hat{\sigma}(Q \bar{Q}(n)+X)$ is the perturbative cross section of the $Q \bar{Q}$ pair in the color and angular momentum state $n, \mathscr{O}_{n}^{H}$ is the corresponding NRQCD operator. The matrix elements $\left\langle 0\left|\mathscr{O}_{n}^{H}\right| 0\right\rangle$ have known scalings in $v$, the heavy quark velocity inside the quarkonium $\left(v^{2} \approx 0.3\right.$ for charmonia, $v^{2} \approx 0.1$ for bottomonia). Hence, the sum in Eq. (3.1) is organized in powers of $v$, and in practice, the sum is truncated at a desired accuracy.

The NRQCD long-distance matrix elements (LDMEs) in Eq. (3.1) are nonperturbative objects. In some cases, where the operator creates a $Q \bar{Q}$ in a color singlet state, the LDMEs can be computed in potential models [15], which are in general good agreement with lattice QCD calculations [16]. 
However, except for some limited cases at lowest orders in $v$, it is not known how to compute the LDMEs from first principles. Especially, when the NRQCD operator creates a $Q \bar{Q}$ in a color octet state, it is not possible to describe the evolution of a color-octet $Q \bar{Q}$ to a color-singlet meson using potentials. In phenomenological studies, these color octet LDMEs are obtained by comparing Eq. (3.1) with data.

It is well known that at large $p_{T}$, calculations based on Eq. (3.1) at leading order in $v$ can give unsatisfactory description of data. In case of production in hadron colliders, contributions at higher orders in $v$ can be important, because the $Q \bar{Q}$ in color octet states can be produced with less powers of $\alpha_{s}$ than the color singlet channel [17]. In present-day heavy-quarkonium-production phenomenology, Eq. (3.1) is usually truncated at relative order $v^{4}$. In recent years, the perturbative cross sections have been calculated to NLO in $\alpha_{s}$. In case of $J / \psi$ production, phenomenological determinations of color-octet LDMEs depend strongly on the choice of data [18, 19, 20, 21]. Especially, while LDMEs determined from high- $p_{T}$ cross section data $\left(p_{T} \gg m_{\text {Quarkonium }}\right)$ lead to predictions of $J / \psi$ polarization $[19,18,21]$ and $J / \psi$ momentum distribution in jet [22] that are in reasonable agreement with data $[23,24,25,26,27,28]$, they are often at odds with measurements at small $p_{T}$, including data from lepton colliders [29], photoproduction [30, 31, 32], and $\eta_{c}$ production [33]. Although LDME determinations including low- $p_{T}$ data can describe some, though not all, of the low- $p_{T}$ measurements [20], such determinations tend to disagree with high- $p_{T}$ data. An efficient way to resolve such issues might be to find a way to constrain color-octet LDMEs from first principles. For example, the color-octet decay LDMEs can be computed using the potential NRQCD effective field theory [34, 35].

\subsection{Color Singlet Model}

The color-singlet model (CSM) [36, 37, 38] is one of the oldest models of heavy quarkonium production. In CSM, the production rate is given by

$$
\sigma(H+X)=\hat{\sigma}\left(Q \bar{Q}_{\text {color singlet }}+X\right)\left\langle 0\left|\mathscr{O}^{H}\right| 0\right\rangle,
$$

where $\hat{\sigma}\left(Q \bar{Q}_{\text {color singlet }}+X\right)$ is the perturbative cross section of a color-singlet $Q \bar{Q}$ pair with the same $J^{P C}$ of the hadron $H$, and $\left\langle 0\left|\mathscr{O}^{H}\right| 0\right\rangle$ is the color-singlet LDME. Since color-singlet LDMEs can be obtained from potential models, there are no free adjustable parameters in CSM. For some kinds of quarkonia, such as $J / \psi$ and $\eta_{c}$, Eq. (3.1) at leading order in $v$ gives Eq. (3.2). This is not true in general; for example, the production of $P$-wave quarkonia such as the $\chi_{c J}$ involves both the color-singlet and color-octet LDMEs, which are necessary for the cancellation of infrared divergences in the perturbative cross sections in Eq. (3.1); on the other hand, direct application of Eq. (3.2) to $P$-wave quarkonium leads to an infrared divergent result [14]. This is because CSM does not correctly incorporate the soft degrees of freedom in QCD. CSM predictions of $J / \psi$ cross sections are NRQCD predictions at leading order in $v$. These predictions are well known to disagree with both cross sections and polarization [20].

\subsection{Color Evaporation Model}

Color evaporation model (CEM) is an opposite extreme compared to CSM where $Q \bar{Q}$ in any color, spin and angular momentum state can contribute to the cross section, as long as the invariant 
mass of the pair is smaller than twice the mass of $D$ mesons, and the hadronization is described by a universal constant per quarkonium state $[39,40]$. While this approach is compatible with quarkhadron duality, the constant factor that describes hadronization has no origin from QCD. CEM is also at odds with NRQCD, since the constant hadronization factor implies that all NRQCD LDMEs scale the same way [41]. Nevertheless, CEM can produce results that agree well with data [42, 43].

\section{Summary}

In this paper, we reviewed the current status of heavy flavor and heavy quarkonium production phenomenology. The production mechanism of heavy flavored mesons is in good theoretical control, making them suitable to be used as tools to probe QCD. In contrast, heavy quarkonium production mechanism is not understood well in effective field theory methods, and model calculations are often employed. More investigation of the effective field theory method would be necessary to understand heavy quarkonium production mechanisms from first principles.

\section{References}

[1] J. C. Collins, D. E. Soper and G. F. Sterman, Phys. Lett. 134B, 263 (1984).

[2] G. T. Bodwin, Phys. Rev. D 31, 2616 (1985) Erratum: [Phys. Rev. D 34, 3932 (1986)].

[3] J. C. Collins, D. E. Soper and G. F. Sterman, Adv. Ser. Direct. High Energy Phys. 5, 1 (1989) [hep-ph/0409313].

[4] J. Collins, Int. J. Mod. Phys. Conf. Ser. 25, 1460001 (2014) [arXiv:1307.2920 [hep-ph]].

[5] M. A. G. Aivazis, J. C. Collins, F. I. Olness and W. K. Tung, Phys. Rev. D 50, 3102 (1994) [hep-ph/9312319].

[6] J. C. Collins and D. E. Soper, Nucl. Phys. B 194, 445 (1982).

[7] M. Cacciari, M. Greco and P. Nason, JHEP 9805, 007 (1998) [hep-ph/9803400].

[8] M. Cacciari, S. Frixione and P. Nason, JHEP 0103, 006 (2001) [hep-ph/0102134].

[9] B. A. Kniehl, G. Kramer, I. Schienbein and H. Spiesberger, Phys. Rev. D 71, 014018 (2005) [hep-ph/0410289].

[10] B. A. Kniehl, G. Kramer, I. Schienbein and H. Spiesberger, Eur. Phys. J. C 72, 2082 (2012) [arXiv:1202.0439 [hep-ph]].

[11] M. Cacciari, S. Frixione, N. Houdeau, M. L. Mangano, P. Nason and G. Ridolfi, JHEP 1210, 137 (2012) [arXiv:1205.6344 [hep-ph]].

[12] R. Maciuła, A. Szczurek and A. Cisek, Phys. Rev. D 99, no. 5, 054014 (2019) [arXiv:1810.08063 [hep-ph]].

[13] R. Aaij et al. [LHCb Collaboration], Phys. Rev. D 99, no. 5, 052011 (2019) [arXiv:1902.05599 [hep-ex]].

[14] G. T. Bodwin, E. Braaten and G. P. Lepage, Phys. Rev. D 51, 1125 (1995) Erratum: [Phys. Rev. D 55, 5853 (1997)] [hep-ph/9407339].

[15] N. Brambilla, A. Pineda, J. Soto and A. Vairo, Nucl. Phys. B 566, 275 (2000) [hep-ph/9907240]. 
[16] G. T. Bodwin, D. K. Sinclair and S. Kim, Phys. Rev. Lett. 77, 2376 (1996) [hep-lat/9605023].

[17] P. L. Cho and A. K. Leibovich, Phys. Rev. D 53, 150 (1996) [hep-ph/9505329].

[18] K.-T. Chao, Y.-Q. Ma, H.-S. Shao, K. Wang, and Y.-J. Zhang, Phys. Rev. Lett. 108, 242004 (2012) [arXiv:1201.2675 [hep-ph]].

[19] B. Gong, L.-P. Wan, J.-X. Wang, and H.-F. Zhang, Phys. Rev. Lett. 110, 042002 (2013) [arXiv:1205.6682 [hep-ph]].

[20] M. Butenschoen and B. A. Kniehl, Phys. Rev. D 84, 051501 (2011) [arXiv:1105.0820 [hep-ph]].

[21] G. T. Bodwin, K. T. Chao, H. S. Chung, U. R. Kim, J. Lee and Y. Q. Ma, Phys. Rev. D 93, no. 3, 034041 (2016) [arXiv:1509.07904 [hep-ph]].

[22] R. Bain, L. Dai, A. Leibovich, Y. Makris and T. Mehen, Phys. Rev. Lett. 119, no. 3, 032002 (2017) [arXiv:1702.05525 [hep-ph]].

[23] B. Abelev et al. [ALICE Collaboration], Phys. Rev. Lett. 108, 082001 (2012) [arXiv:1111.1630 [hep-ex]].

[24] S. Acharya et al. [ALICE Collaboration], Eur. Phys. J. C 78, no. 7, 562 (2018) [arXiv:1805.04374 [hep-ex]].

[25] S. Chatrchyan et al. [CMS Collaboration], Phys. Lett. B 727, 381 (2013) [arXiv:1307.6070 [hep-ex]].

[26] R. Aaij et al. [LHCb Collaboration], Eur. Phys. J. C 73, no. 11, 2631 (2013) [arXiv:1307.6379 [hep-ex]].

[27] R. Aaij et al. [LHCb Collaboration], Phys. Rev. Lett. 118, no. 19, 192001 (2017) [arXiv:1701.05116 [hep-ex]].

[28] CMS Collaboration [CMS Collaboration], CMS-PAS-HIN-18-012.

[29] J. Abdallah et al. [DELPHI Collaboration], Phys. Lett. B 565, 76 (2003) [hep-ex/0307049].

[30] S. Chekanov et al. [ZEUS Collaboration], Eur. Phys. J. C 27 (2003) 173 [hep-ex/0211011].

[31] C. Adloff et al. [H1 Collaboration], Eur. Phys. J. C 25, 25 (2002) [hep-ex/0205064].

[32] F. D. Aaron et al. [H1 Collaboration], Eur. Phys. J. C 68, 401 (2010) [arXiv:1002.0234 [hep-ex]].

[33] R. Aaij et al. [LHCb Collaboration], Eur. Phys. J. C 75, no. 7, 311 (2015) [arXiv:1409.3612 [hep-ex]].

[34] N. Brambilla, D. Eiras, A. Pineda, J. Soto and A. Vairo, Phys. Rev. Lett. 88, 012003 (2002) [hep-ph/0109130].

[35] N. Brambilla, D. Eiras, A. Pineda, J. Soto and A. Vairo, Phys. Rev. D 67, 034018 (2003) [hep-ph/0208019].

[36] S. D. Ellis, M. B. Einhorn and C. Quigg, Phys. Rev. Lett. 36, 1263 (1976).

[37] C. E. Carlson and R. Suaya, Phys. Rev. D 14, 3115 (1976).

[38] C. H. Chang, Nucl. Phys. B 172, 425 (1980).

[39] H. Fritzsch, Phys. Lett. 67B, 217 (1977).

[40] F. Halzen, Phys. Lett. 69B, 105 (1977).

[41] G. T. Bodwin, E. Braaten and J. Lee, Phys. Rev. D 72, 014004 (2005) [hep-ph/0504014].

[42] Y. Q. Ma and R. Vogt, Phys. Rev. D 94, no. 11, 114029 (2016) [arXiv:1609.06042 [hep-ph]].

[43] V. Cheung and R. Vogt, Phys. Rev. D 99, no. 3, 034007 (2019) [arXiv:1811.11570 [hep-ph]]. 